\title{
THE EFFECTS OF AGING, ARTERIOSCLEROSIS, AND HYPERTENSION UPON THE CEREBRAL CIRCULATION ${ }^{1}$
}

\author{
By HENRY A. SHENKIN, PAUL NOVAK,² BERNARD GOLUBOFF, ALVIN M. SOFFE, \\ AND LEONARD BORTIN WITH THE TECHNICAL ASSISTANCE OF DORIS GOLDEN \\ AND MRS. PETER BATSON
}

\begin{abstract}
(From the Departments of Neurosurgery, Research, and Medicine of the Albert Einstein Medical Center, Southern Division, Philadelphia, Penna.)
\end{abstract}

(Submitted for publication November 10, 1952; accepted February 11, 1953)

Various investigators have applied the nitrous oxide method (1) to the study of the effects of aging, vascular sclerosis, and hypertension upon the cerebral blood flow and metabolism. Freyhan, Woodford, and Kety (2) measured the cerebral blood flow in cases manifesting psychoses secondary to advanced cerebral arteriosclerosis. Significant reductions of cerebral blood flow and oxygen utilization were noted. Scheinberg (3) reported reduction of both cerebral blood flow and oxygen consumption in patients with histories of previous cerebrovascular accidents. Greater reductions in these measurements were noted in patients who exhibited mental aberrations secondary to cerebrovascular disease. Fazekas, Alman, and Bessman (4) found a reduction of the cerebral blood flow and oxygen consumption in individuals above 50 years of age with no clinical evidence of cerebrovascular disease. Kety, Hafkenschiel, Jeffers, Leopold, and Shenkin (5) demonstrated the presence of a normal cerebral blood flow and oxygen consumption and an elevated cerebrovascular resistance in a series of cases of essential hypertension. The present investigation is an attempt to clarify and extend knowledge concerning the interrelated effects of aging, hypertension, and arteriosclerosis upon the cerebral hemodynamics and metabolism.

\section{METHODS}

The cerebral blood flow (CBF) was measured by the nitrous oxide technique (1). Patients were in the postabsorptive state and the recumbent position. The jugular venous pressure (JVP) was measured with a spinal fluid manometer using the level at which the vessel was entered as the " $O$ " reference point. This is generally at the right atrial level in the supine position. The mean

1 Supported by a grant from the National Institutes of Health of the United States Public Health Service.

2 National Heart Institute Postdoctorate Research Fellow, 1952-53. arterial blood pressure (MABP) was measured in a femoral artery with a damped mercury manometer. The oxygen and carbon dioxide contents of the blood were determined by the method of Van Slyke and Neill (6). The blood $\mathrm{pH}$ was determined anaerobically at room temperature with a glass electrode and corrected to body temperature using the formula of Rosenthal (7). The $\mathrm{pCO}_{2}$ was determined from standard nomograms (6).

\section{MATERIAL}

The subjects have been divided into five groups: 1) Normotensive individuals under 40 years of age with no clinical evidence of arteriosclerosis. 2) Normotensive individuals above 50 years of age with systemic or cerebral vascular disease. Systemic arteriosclerosis was diagnosed by the presence of vascular calcifications, signs and symptoms of coronary artery disease, or peripheral vascular impairment. No attempt was made to further quantitate the severity of the arteriosclerosis. A mean arterial blood pressure of $115 \mathrm{~mm}$. $\mathrm{Hg}$ has been arbitrarily used to separate the hypertensive from the normotensive. Cerebrovascular disease was considered present where a history of a past cerebrovascular accident was obtained. 3) Hypertensive arteriosclerotic individuals above 40 years of age without a history of a cerebrovascular accident or mental deterioration. 4) Hypertensive arteriosclerotic individuals above 40 years of age with a history of cerebrovascular accidents or mental deterioration. 5) Hypertensive individuals with no evidence of vascular disease. Retinopathy was not more severe than grade II.

\section{RESULTS}

Tables Ia and Ib summarize clinical data and the values obtained for the various cerebral circulatory functions and blood constituents of Group 1. The mean values are comparable to normals established by others (1).

Tables IIa and IIb present the clinical, cerebral circulatory, and blood constituent data for Group 2. The mean CBF of $46 \mathrm{cc}$. per $100 \mathrm{gm}$. per min. is not significantly below the value noted for Group $1(\mathrm{p}>.2)$. The CVR of $2.1 \mathrm{~mm}$. Hg per cc. per $100 \mathrm{gm}$. per min. is similarly higher, but not sig- 
TABLE Ia

Group 1: Clinical and conebral circulatory data*

\begin{tabular}{|c|c|c|c|c|c|c|c|c|c|}
\hline Subject & Age & Sex & Diagnosis & MABP & JVP & CBF & CVR & $\mathrm{CMRO}_{2}$ & R.Q \\
\hline $\begin{array}{l}\text { G. C. } \\
\text { S. W. } \\
\text { F. B. } \\
\text { S. G. } \\
\text { M. H. } \\
\text { D. M. } \\
\text { S. R. } \\
\text { A. H. } \\
\text { S. K. } \\
\text { E. G. } \\
\text { S. D. } \\
\text { D. O. }\end{array}$ & $\begin{array}{l}40 \\
40 \\
36 \\
25 \\
18 \\
31 \\
36 \\
29 \\
26 \\
26 \\
23 \\
27\end{array}$ & $\begin{array}{l}\mathbf{M} \\
\mathbf{F} \\
\mathbf{F} \\
\mathbf{F} \\
\mathbf{M} \\
\mathbf{F} \\
\mathbf{F} \\
\mathbf{M} \\
\mathbf{F} \\
\mathbf{F} \\
\mathbf{F}\end{array}$ & $\begin{array}{l}\text { Duodenal ulcer } \\
\text { Psychoneurosis } \\
\text { Trigeminal neuralgia } \\
\text { Headache, functional } \\
\text { Headache, functional } \\
\text { Resp. infection } \\
\text { Functional G.I. } \\
\text { Functional G.I. } \\
\text { Psychoneurosis } \\
\text { Migraine } \\
\text { Neurodermatitis } \\
\text { Post op., mastoidect. }\end{array}$ & $\begin{array}{r}99 \\
84 \\
79 \\
81 \\
91 \\
79 \\
100 \\
111 \\
95 \\
100 \\
91 \\
83\end{array}$ & $\begin{array}{r}186 \\
90 \\
101 \\
98 \\
84 \\
98 \\
73 \\
61 \\
152 \\
108 \\
101 \\
80\end{array}$ & $\begin{array}{l}47 \\
77 \\
47 \\
35 \\
22 \\
54 \\
38 \\
52 \\
74 \\
54 \\
78 \\
57\end{array}$ & $\begin{array}{l}1.8 \\
1.0 \\
1.5 \\
2.1 \\
3.9 \\
1.3 \\
2.5 \\
2.0 \\
1.1 \\
1.7 \\
1.1 \\
1.3\end{array}$ & $\begin{array}{l}2.8 \\
4.2 \\
3.4 \\
2.5 \\
1.6 \\
2.9 \\
2.0 \\
4.8 \\
4.9 \\
2.7 \\
5.1 \\
3.8\end{array}$ & $\begin{array}{l}1.00 \\
0.93 \\
0.88 \\
0.97 \\
0.99 \\
1.29 \\
0.78 \\
1.06 \\
1.01 \\
0.92 \\
0.92 \\
0.98\end{array}$ \\
\hline $\begin{array}{l}\text { Mean } \\
\text { S.E. }\end{array}$ & 30 & & & $\begin{array}{r}91 \\
\pm 3\end{array}$ & $\begin{array}{r}103 \\
\pm 10\end{array}$ & $\begin{array}{r}53 \\
\pm 5\end{array}$ & $\begin{array}{r}1.8 \\
\pm 0.5\end{array}$ & $\begin{array}{r}3.4 \\
\pm 0.6\end{array}$ & $\begin{array}{r}0.98 \\
\pm 0.01\end{array}$ \\
\hline
\end{tabular}

* MABP: Mean arterial blood pressure, mm. Hg.

JVP: Jugular venous pressure, $\mathrm{mm} . \mathrm{H}_{2} \mathrm{O}$.

CBF: Cerebral blood flow, cc. per $100 \mathrm{gm}$. per min.

CVR: Cerebrovascular resistance, $\mathrm{mm}$. $\mathrm{Hg}$ per cc. per $100 \mathrm{gm}$. per min.

$\mathrm{CMRO}_{2}$ : Cerebral oxygen consumption, cc. per $100 \mathrm{gm}$. per min.

R.Q.: Cerebral respiratory quotient.

S.E.: Standard error.

nificantly so, than that for Group $1(p>.5)$. The other cerebral circulatory measurements do not differ significantly from those presented for Group 1. The mean values for the various blood constituents listed fall within the normal range.

Table III lists the cerebral circulatory data for the individuals in Group 3, the hypertensive arteriosclerotic patients without previous vascular accidents or mental deterioration. The mean CBF of $39 \mathrm{cc}$. per $100 \mathrm{gm}$. per min. is 26 per cent below the normal of $53 \mathrm{cc}$. per $100 \mathrm{gm}$. per min. The mean CVR of $3.4 \mathrm{~mm}$. $\mathrm{Hg}$ per cc. per $100 \mathrm{gm}$. per min. per cc. is 89 per cent higher than the value noted for Group 1. The mean $\mathrm{CMRO}_{2}$ of $2.4 \mathrm{cc}$. per $100 \mathrm{gm}$. per min. is 27 per cent below the value recorded for Group 1. These values also differ significantly from the corresponding values of Group 2.

Table IVa presents the cerebral circulatory data for Group 4, patients similar to those of Group 3

TABLE Ib

Group 1: Blood constituents*

\begin{tabular}{|c|c|c|c|c|c|c|c|c|c|c|}
\hline Subject & $\mathrm{AO}_{2}$ & $\mathrm{ACO}_{2}$ & $\mathrm{ApH}$ & $\mathrm{ApCO}_{2}$ & $\mathrm{VO}_{2}$ & $\mathrm{VCO}_{2}$ & $\mathrm{VpH}$ & $\mathrm{VpCO}_{2}$ & $(A-V) O_{2}$ & $(\mathrm{~A}-\mathrm{V}) \mathrm{CO}_{2}$ \\
\hline $\begin{array}{l}\text { G. C. } \\
\text { S. W. } \\
\text { F. B. } \\
\text { S. G. } \\
\text { M. H. } \\
\text { D. M. } \\
\text { S. R. } \\
\text { A. H. } \\
\text { S. K. } \\
\text { E. G. } \\
\text { S. D. } \\
\text { D. O. }\end{array}$ & $\begin{array}{l}16.8 \\
14.9 \\
14.6 \\
19.4 \\
17.9 \\
18.8 \\
16.5 \\
15.4 \\
20.2 \\
15.0 \\
11.9 \\
12.1\end{array}$ & $\begin{array}{l}47.3 \\
54.4 \\
40.1 \\
40.8 \\
39.6 \\
46.8 \\
51.4 \\
46.6 \\
50.9 \\
50.9 \\
48.5 \\
48.2\end{array}$ & $\begin{array}{l}7.33 \\
7.33 \\
7.31 \\
7.36 \\
7.42 \\
7.29 \\
7.34 \\
7.39 \\
7.39 \\
7.33 \\
7.38 \\
7.41\end{array}$ & $\begin{array}{l}46 \\
51 \\
39 \\
38 \\
32 \\
47 \\
48 \\
40 \\
45 \\
48 \\
42 \\
37\end{array}$ & \begin{tabular}{r|}
10.8 \\
9.4 \\
7.3 \\
12.4 \\
10.6 \\
12.3 \\
11.3 \\
6.3 \\
13.5 \\
10.5 \\
10.0 \\
5.3 \\
5.4
\end{tabular} & $\begin{array}{l}\mathbf{5 3 . 3} \\
59.4 \\
46.6 \\
47.7 \\
46.9 \\
55.3 \\
55.4 \\
56.3 \\
57.6 \\
55.5 \\
54.5 \\
54.7\end{array}$ & $\begin{array}{l}7.30 \\
7.30 \\
7.28 \\
7.33 \\
7.39 \\
7.28 \\
7.29 \\
7.39 \\
7.33 \\
7.29 \\
7.32 \\
7.35\end{array}$ & $\begin{array}{l}51 \\
55 \\
47 \\
40 \\
36 \\
57 \\
57 \\
48 \\
51 \\
54 \\
49 \\
46\end{array}$ & $\begin{array}{l}6.0 \\
5.4 \\
7.3 \\
7.1 \\
7.3 \\
6.6 \\
5.2 \\
9.1 \\
6.6 \\
5.0 \\
6.6 \\
6.7\end{array}$ & $\begin{array}{l}6.0 \\
5.0 \\
6.4 \\
6.8 \\
7.3 \\
8.5 \\
4.1 \\
9.7 \\
6.7 \\
4.6 \\
6.0 \\
6.5\end{array}$ \\
\hline $\begin{array}{l}\text { Mean } \\
\text { S.E. }\end{array}$ & $\begin{array}{r}16.1 \\
\pm 0.8\end{array}$ & $\begin{array}{r}47.1 \\
\pm 1.4\end{array}$ & $\begin{array}{r}7.36 \\
\pm .02\end{array}$ & $\begin{array}{r}43 \\
\pm 2\end{array}$ & $\begin{array}{r}9.6 \\
\pm 0.8\end{array}$ & $\begin{array}{r}53.6 \\
\pm 1.2\end{array}$ & $\begin{array}{r}7.32 \\
\pm .01\end{array}$ & $\begin{array}{r}49 \\
\pm 2\end{array}$ & $\begin{array}{r}6.6 \\
\pm 0.3\end{array}$ & $\begin{array}{r}6.5 \\
\pm 0.5\end{array}$ \\
\hline
\end{tabular}

* A: Arterial.

V: Venous (jugular).

$\mathrm{O}_{2}$ : Oxygen content, vol. per cent.

$\mathrm{CO}_{2}$ : Carbon dioxide content, vol. per cent.

S.E.: Standard error. 
TABLE IIa

Group 2: Clinical and cerebral circulatory data*

\begin{tabular}{|c|c|c|c|c|c|c|c|c|c|}
\hline Subject & Age & Sex & Diagnoeis & MABP & JVP & CBF & $\mathrm{CMRO}_{2}$ & CVR & R.Q. \\
\hline $\begin{array}{l}\text { M.D. } \\
\text { S.A. } \\
\text { H.S. } \\
\text { J.P. } \\
\text { E. G. } \\
\text { B. D. } \\
\text { W.H. } \\
\text { E.L. } \\
\text { E.G. } \\
\text { M.S. } \\
\text { W.F. } \\
\text { M.So. } \\
\text { H. K. } \\
\text { F. F. } \\
\text { B. B. } \\
\text { S. K. } \\
\text { J.L. } \\
\text { A. F. } \\
\text { C. Z. } \\
\text { J.S. } \\
\text { A.Di. } \\
\text { I. K. } \\
\text { T. B. }\end{array}$ & $\begin{array}{l}59 \\
64 \\
75 \\
70 \\
56 \\
68 \\
55 \\
66 \\
55 \\
62 \\
73 \\
65 \\
65 \\
75 \\
75 \\
58 \\
76 \\
80 \\
86 \\
45 \\
61 \\
69 \\
69\end{array}$ & $\begin{array}{l}\mathbf{F} \\
\mathbf{M} \\
\mathbf{M} \\
\mathbf{M} \\
\mathbf{F} \\
\mathbf{M} \\
\mathbf{M} \\
\mathbf{M} \\
\mathbf{M} \\
\mathbf{M} \\
\mathbf{M} \\
\mathbf{M} \\
\mathbf{M} \\
\mathbf{M} \\
\mathbf{F} \\
\mathbf{F} \\
\mathbf{M} \\
\mathbf{M} \\
\mathbf{F} \\
\mathbf{M} \\
\mathbf{M} \\
\mathbf{M} \\
\mathbf{F}\end{array}$ & $\begin{array}{l}\text { Functional G.I. } \\
\text { Duodenal ulcer } \\
\text { Chronic cholecystitis } \\
\text { Acute gastritis } \\
\text { Old CVA } \\
\text { Headache, functional } \\
\text { Old CVA } \\
\text { Bronchiogenic Ca } \\
\text { Pituitary tumor } \\
\text { ASCVD } \\
\text { Pancreatic Ca } \\
\text { Rheumatoid arthritis } \\
\text { Multiple myeloma } \\
\text { ASCVD } \\
\text { Old CVA } \\
\text { Headache, functional } \\
\text { Viral pneumonitis } \\
\text { ASCVD } \\
\text { ASCVD } \\
\text { ASCVD } \\
\text { G.I. malignancy } \\
\text { ASCVD } \\
\text { ASCVD }\end{array}$ & $\begin{array}{r}100 \\
99 \\
100 \\
77 \\
98 \\
95 \\
100 \\
91 \\
86 \\
100 \\
107 \\
102 \\
93 \\
88 \\
90 \\
72 \\
115 \\
98 \\
105 \\
105 \\
96 \\
87 \\
88\end{array}$ & $\begin{array}{r}65 \\
75 \\
38 \\
59 \\
98 \\
156 \\
107 \\
90 \\
77 \\
108 \\
90 \\
82 \\
82 \\
80 \\
54 \\
60 \\
39 \\
90 \\
116 \\
74 \\
88 \\
108\end{array}$ & $\begin{array}{l}48 \\
29 \\
38 \\
98 \\
54 \\
45 \\
41 \\
80 \\
33 \\
36 \\
48 \\
40 \\
54 \\
51 \\
32 \\
51 \\
42 \\
41 \\
37 \\
41 \\
45 \\
40 \\
30\end{array}$ & $\begin{array}{l}3.3 \\
1.5 \\
3.0 \\
6.4 \\
3.9 \\
2.8 \\
2.8 \\
3.1 \\
1.0 \\
2.2 \\
3.2 \\
2.4 \\
2.9 \\
2.7 \\
2.0 \\
3.5 \\
3.3 \\
2.3 \\
1.0 \\
2.2 \\
2.7 \\
2.1 \\
1.7\end{array}$ & $\begin{array}{l}2.0 \\
3.2 \\
2.6 \\
0.8 \\
1.7 \\
1.8 \\
2.2 \\
1.1 \\
2.4 \\
2.6 \\
2.1 \\
2.4 \\
1.6 \\
.1 .6 \\
2.6 \\
1.3 \\
2.7 \\
2.3 \\
2.7 \\
2.3 \\
2.0 \\
2.1 \\
2.7\end{array}$ & $\begin{array}{l}0.89 \\
1.12 \\
1.01 \\
0.51 \\
0.92 \\
0.81 \\
0.96 \\
0.68 \\
0.83 \\
0.97 \\
0.80 \\
1.00 \\
0.97 \\
0.91 \\
1.19 \\
0.96 \\
0.99 \\
1.12 \\
0.84 \\
1.07 \\
0.83 \\
0.71 \\
0.95\end{array}$ \\
\hline $\begin{array}{l}\text { Mean } \\
\text { S.E. }\end{array}$ & 68 & & & $\begin{array}{r}95 \\
\pm 2\end{array}$ & $\begin{array}{r}83 \\
\pm 6\end{array}$ & $\begin{array}{r}46 \\
\pm 3\end{array}$ & $\begin{array}{r}2.7 \\
\pm 0.2\end{array}$ & $\begin{array}{r}2.1 \\
\pm 0.1\end{array}$ & $\begin{array}{r}0.88 \\
\pm 0.05\end{array}$ \\
\hline
\end{tabular}

* See footnote Table Ia.

TABLE IIb

Group 2: Blood constituents*

\begin{tabular}{|c|c|c|c|c|c|c|c|c|c|c|}
\hline Subject & $\mathrm{AO}_{2}$ & $\mathrm{ACO}_{2}$ & ApH & $\mathrm{ApCO}_{2}$ & $\mathrm{VO}_{2}$ & $\mathrm{vCO}_{2}$ & $\mathrm{VpH}$ & $\mathrm{VpCO}_{2}$ & $(A-V) O_{2}$ & $(A-V) C O$ \\
\hline $\begin{array}{l}\text { M. D. } \\
\text { S. A. } \\
\text { H. S. } \\
\text { J. P. } \\
\text { E. G. } \\
\text { B. D. } \\
\text { W. H. } \\
\text { E. L. } \\
\text { E. G. } \\
\text { M. S. } \\
\text { W. F. } \\
\text { M. S. } \\
\text { H. K. } \\
\text { F. F. } \\
\text { B. B. } \\
\text { S. K. } \\
\text { J. L. } \\
\text { A. F. } \\
\text { C. Z. } \\
\text { J. S. } \\
\text { A. D. } \\
\text { I. K. } \\
\text { T. B. }\end{array}$ & $\begin{array}{r}18.5 \\
18.4 \\
17.0 \\
15.1 \\
19.8 \\
18.1 \\
16.4 \\
9.9 \\
19.5 \\
19.2 \\
19.7 \\
16.4 \\
13.8 \\
12.1 \\
15.0 \\
16.7 \\
16.5 \\
14.5 \\
16.1 \\
18.9 \\
17.8 \\
18.7 \\
17.6\end{array}$ & $\begin{array}{l}48.1 \\
44.8 \\
60.7 \\
50.0 \\
46.3 \\
43.6 \\
49.0 \\
48.6 \\
42.5 \\
48.6 \\
52.0 \\
51.3 \\
32.3 \\
42.0 \\
44.9 \\
47.4 \\
44.5 \\
49.0 \\
50.2 \\
47.5 \\
47.7 \\
51.1 \\
50.3\end{array}$ & $\begin{array}{l}7.31 \\
7.34 \\
7.35 \\
\overline{7.36} \\
\overline{7.37} \\
7.45 \\
\overline{7.31} \\
7.33 \\
= \\
= \\
\overline{-} \\
7.33 \\
7.39 \\
7.35 \\
7.37 \\
7.35 \\
7.36 \\
7.37 \\
7.36\end{array}$ & $\begin{array}{l}47 \\
43 \\
56 \\
43 \\
42 \\
34 \\
49 \\
51 \\
- \\
- \\
45 \\
38 \\
44 \\
44 \\
43 \\
43 \\
47 \\
45\end{array}$ & $\begin{array}{r}11.6 \\
13.4 \\
9.1 \\
8.6 \\
12.6 \\
12.0 \\
9.7 \\
6.0 \\
16.6 \\
13.5 \\
12.9 \\
10.3 \\
8.5 \\
6.9 \\
8.7 \\
9.9 \\
8.8 \\
8.8 \\
13.3 \\
13.6 \\
11.7 \\
13.3 \\
11.8\end{array}$ & $\begin{array}{l}\mathbf{5 4 . 3} \\
\mathbf{5 0 . 4} \\
\mathbf{6 8 . 6} \\
\mathbf{5 3 . 3} \\
\mathbf{5 2 . 3} \\
\mathbf{4 8 . 5} \\
\mathbf{5 5 . 4} \\
\mathbf{5 1 . 3} \\
44.8 \\
\mathbf{5 4 . 5} \\
\mathbf{5 7 . 3} \\
\mathbf{5 7 . 3} \\
\mathbf{3 7 . 5} \\
\mathbf{4 7 . 5} \\
\mathbf{5 2 . 4} \\
\mathbf{5 3 . 9} \\
\mathbf{5 2 . 1} \\
\mathbf{5 5 . 4} \\
\mathbf{5 2 . 5} \\
\mathbf{5 3 . 1} \\
\mathbf{5 2 . 8} \\
\mathbf{5 4 . 8} \\
\mathbf{5 5 . 8}\end{array}$ & $\begin{array}{l}7.27 \\
7.30 \\
7.30 \\
7.30 \\
\overline{7.31} \\
7.36 \\
\overline{7.29} \\
7.27 \\
= \\
- \\
\overline{7} \\
7.31 \\
7.33 \\
7.32 \\
7.34 \\
7.31 \\
7.29 \\
7.35 \\
7.32\end{array}$ & $\begin{array}{l}55 \\
53 \\
70 \\
\frac{47}{77} \\
51 \\
41 \\
57 \\
60 \\
- \\
- \\
53 \\
46 \\
53 \\
50 \\
53 \\
55 \\
49 \\
52\end{array}$ & $\begin{array}{l}6.9 \\
5.0 \\
7.8 \\
6.5 \\
7.2 \\
6.1 \\
6.8 \\
3.9 \\
2.9 \\
5.7 \\
6.8 \\
6.0 \\
5.3 \\
5.2 \\
6.3 \\
6.8 \\
7.7 \\
5.7 \\
2.8 \\
5.2 \\
5.1 \\
5.3 \\
5.8\end{array}$ & $\begin{array}{l}6.2 \\
5.6 \\
7.9 \\
3.3 \\
6.6 \\
4.9 \\
6.5 \\
2.6 \\
2.4 \\
5.9 \\
5.4 \\
6.0 \\
5.1 \\
4.8 \\
7.5 \\
6.5 \\
7.6 \\
6.4 \\
2.3 \\
5.6 \\
6.1 \\
3.7 \\
5.5\end{array}$ \\
\hline $\begin{array}{l}\text { Mean } \\
\text { S.E. }\end{array}$ & $\begin{array}{r}16.8 \\
\pm 0.5\end{array}$ & $\begin{array}{r}47.5 \\
\pm 1.1\end{array}$ & $\begin{array}{r}7.36 \\
\pm .01\end{array}$ & $\begin{array}{r}45 \\
\pm 1\end{array}$ & $\begin{array}{r}10.9 \\
\pm 0.5\end{array}$ & $\begin{array}{r}52.9 \\
\pm 1.2\end{array}$ & $\begin{array}{r}7.31 \\
\pm .02\end{array}$ & $\begin{array}{r}53 \\
\pm 2\end{array}$ & $\begin{array}{r}5.8 \\
\pm 0.3\end{array}$ & $\begin{array}{r}5.4 \\
\pm 0.3\end{array}$ \\
\hline
\end{tabular}

* See footnote Table Ib. 
TABLE III

Group 3: Clinical and cerebral circulatory data*

\begin{tabular}{|c|c|c|c|c|c|c|c|c|c|}
\hline Subject & Age & Sex & Diagnosis & MABP & JVP & $\mathrm{CBF}$ & CVR & $\mathrm{CMRO}_{2}$ & R.Q. \\
\hline $\begin{array}{l}\text { M.S. } \\
\text { S. F. } \\
\text { J. L. } \\
\text { A. W. } \\
\text { E. S. } \\
\text { I. B. }\end{array}$ & $\begin{array}{l}68 \\
64 \\
62 \\
62 \\
66 \\
41\end{array}$ & $\begin{array}{l}\mathbf{M} \\
\mathbf{F} \\
\mathbf{M} \\
\mathbf{F} \\
\mathbf{F} \\
\mathbf{F}\end{array}$ & $\begin{array}{l}\text { HASCVD } \\
\text { HASCVD } \\
\text { HASCVD } \\
\text { HASCVD } \\
\text { HASCVD } \\
\text { Pheochromocytoma }\end{array}$ & $\begin{array}{l}120 \\
132 \\
156 \\
125 \\
138 \\
160\end{array}$ & $\begin{array}{r}60 \\
54 \\
78 \\
145 \\
123 \\
108\end{array}$ & $\begin{array}{l}33 \\
33 \\
41 \\
44 \\
39 \\
42\end{array}$ & $\begin{array}{l}3.5 \\
3.9 \\
3.7 \\
2.6 \\
3.3 \\
3.6\end{array}$ & $\begin{array}{l}2.4 \\
2.6 \\
2.7 \\
3.7 \\
1.6 \\
1.6\end{array}$ & $\begin{array}{l}0.98 \\
1.05 \\
0.77 \\
0.92 \\
1.05 \\
1.16\end{array}$ \\
\hline $\begin{array}{l}\text { Mean } \\
\text { S.E. }\end{array}$ & 61 & & & $\begin{array}{l}139 \dagger \\
\pm 7\end{array}$ & $\begin{array}{r}95 \\
\pm 15\end{array}$ & $\begin{array}{c}39 \dagger \\
\pm 2\end{array}$ & $\begin{array}{r}3.4 \dagger \\
\pm 0.2\end{array}$ & $\begin{array}{r}2.4 \\
\pm 0.3\end{array}$ & $\begin{array}{r}0.99 \\
\pm 0.06\end{array}$ \\
\hline
\end{tabular}

* See footnote Table Ia.

† Differs significantly from value for Group 1, p $<.05$.

but with the history of a past cerebrovascular accident or mental deterioration. The values do not differ significantly from those noted for Group 3. Table IV summarizes the blood constituent data for Groups 3 and 4 . No significant variations from normal are observed.

Table Va presents the clinical and cerebral circulatory data for Group 5, cases of essential hypertension uncomplicated by clinically demonstrable arteriosclerosis. All quantities measured, with the exceptions of MABP and CVR, are within normal limits. The high mean JVP, although not significantly different from that noted for the other groups, cannot be explained at present. Variations of the JVP will be discussed in another paper (8). The MABP of $136 \mathrm{~mm}$. $\mathrm{Hg}$ is comparable to values noted for Groups 3 and 4 . The CVR of $2.7 \mathrm{~mm}$. $\mathrm{Hg}$ per cc. per $100 \mathrm{gm}$. per min. is significantly lower than that noted for Groups 3 or 4. Table $\mathrm{Vb}$ presents the data for the blood constituents of Group 5. No significant variations from the normal are observed.

\section{DISCUSSION}

The presence of a CBF and $\mathrm{CMRO}_{2}$, not significantly different from normal in a group whose mean age is 68 , suggests that aging per se has little effect upon these quantities. The failure to obtain results in complete agreement with Fazekas, Alman, and Bessman (4) seems based upon the consideration of the factor of hypertension in the present analysis. In the absence of hypertension, the degree of arteriosclerosis is apparently insufficient to affect significantly the measured cerebral circulatory functions. The wide spread of values in the normotensive arteriosclerotic group and the apparent trend toward a lowered CBF and $\mathrm{CMRO}_{2}$, which could prove significant in a study of a larger group, suggests that hypertension is only one of many contributory factors. However, hypertension emerged from the present series as the one readily definable factor in predicting the occurrence of a reduced $\mathrm{CBF}$ and $\mathrm{CMRO}_{2}$ in arteriosclerotic individuals. Thus, in the hypertensive arteriosclerotic groups the $\mathrm{CBF}$ and

TABLE IVa

Group 4: Clinical and cerebral circulatory data*

\begin{tabular}{|c|c|c|c|c|c|c|c|c|c|}
\hline Subject & Age & Sex & Diagnosis & MABP & JVP & CBF & CVR & $\mathrm{CMRO}_{2}$ & R.Q. \\
\hline $\begin{array}{l}\text { E. S. } \\
\text { S. S. } \\
\text { C. K. } \\
\text { S. G. } \\
\text { S. L. } \\
\text { D. K. } \\
\text { J. S. }\end{array}$ & $\begin{array}{l}45 \\
45 \\
50 \\
51 \\
53 \\
60 \\
57\end{array}$ & $\begin{array}{l}\mathbf{M} \\
\mathbf{F} \\
\mathbf{F} \\
\mathbf{M} \\
\mathbf{M} \\
\mathbf{F} \\
\mathbf{M}\end{array}$ & $\begin{array}{l}\text { Old CVA } \\
\text { Mental deterior. } \\
\text { Old CVA } \\
\text { Old CVA } \\
\text { Old CVA } \\
\text { Mental deterior. } \\
\text { Old CVA }\end{array}$ & $\begin{array}{l}141 \\
140 \\
143 \\
117 \\
123 \\
120 \\
134\end{array}$ & $\begin{array}{r}210 \\
65 \\
35 \\
55 \\
59 \\
100 \\
120\end{array}$ & $\begin{array}{l}51 \\
23 \\
29 \\
34 \\
41 \\
33 \\
48\end{array}$ & $\begin{array}{l}2.5 \\
6.3 \\
4.9 \\
3.3 \\
3.0 \\
3.4 \\
2.7\end{array}$ & $\begin{array}{l}2.6 \\
2.5 \\
2.6 \\
2.1 \\
2.8 \\
2.6 \\
2.7\end{array}$ & $\begin{array}{l}1.27 \\
0.86 \\
0.99 \\
1.11 \\
0.88 \\
0.88 \\
1.08\end{array}$ \\
\hline $\begin{array}{l}\text { Mean } \\
\text { S.E. }\end{array}$ & 51 & & & $\begin{array}{l}131 \\
\pm 4\end{array}$ & $\begin{array}{r}92 \\
\pm 32\end{array}$ & $\begin{array}{l}37 \dagger \\
\pm 4\end{array}$ & $\begin{array}{l}3.7 \dagger \\
\pm 0.5\end{array}$ & $\begin{aligned} & 2.6 \dagger \\
& \pm 0.1\end{aligned}$ & $\begin{array}{r}1.01 \\
\pm 0.07\end{array}$ \\
\hline
\end{tabular}

* See footnote Table Ia.

$\dagger$ Differs significantly from value for Group $1, \mathrm{p}<.05$. 
TABLE IVb

Groups 3 and 4: Blood constituents*

\begin{tabular}{|c|c|c|c|c|c|c|c|c|c|c|}
\hline Subject & $\mathrm{AO}_{2}$ & $\mathrm{ACO}_{2}$ & $\mathrm{ApH}$ & $\mathrm{ApCO}_{2}$ & $\mathrm{VO}_{2}$ & $\mathrm{VCO}_{2}$ & $\mathrm{VpH}$ & $\mathrm{VpCO}_{2}$ & $(A-V) O_{2}$ & $(\mathrm{~A}-\mathrm{V}) \mathrm{CO}_{2}$ \\
\hline $\begin{array}{l}\text { M. S. } \\
\text { S. F. } \\
\text { E. S. } \\
\text { S. S. } \\
\text { C. K. } \\
\text { S. G. } \\
\text { S. L. } \\
\text { D. K. } \\
\text { J. L. } \\
\text { A. W. } \\
\text { J. S. } \\
\text { E. S. } \\
\text { I. B. }\end{array}$ & $\begin{array}{l}21.2 \\
16.8 \\
19.2 \\
22.5 \\
19.1 \\
10.8 \\
19.0 \\
17.4 \\
19.6 \\
19.2 \\
21.2 \\
15.5 \\
13.7\end{array}$ & $\begin{array}{l}40.5 \\
45.6 \\
44.9 \\
54.1 \\
37.8 \\
39.9 \\
48.1 \\
43.8 \\
49.8 \\
49.1 \\
48.2 \\
51.6 \\
59.8\end{array}$ & $\begin{array}{l}7.37 \\
7.30 \\
7.39 \\
7.51 \\
\overline{7.38} \\
7.50 \\
7.43 \\
7.41 \\
7.38 \\
7.34\end{array}$ & $\begin{array}{l}38 \\
47 \\
47 \\
22 \\
\frac{42}{27} \\
40 \\
41 \\
43 \\
\frac{56}{}\end{array}$ & $\begin{array}{r}13.9 \\
8.8 \\
14.1 \\
10.1 \\
10.2 \\
4.7 \\
12.3 \\
9.6 \\
13.0 \\
10.9 \\
15.6 \\
11.4 \\
9.9\end{array}$ & $\begin{array}{l}47.6 \\
53.8 \\
51.3 \\
64.7 \\
46.6 \\
46.6 \\
54.1 \\
50.7 \\
54.9 \\
56.9 \\
54.2 \\
56.0 \\
64.0\end{array}$ & $\begin{array}{l}7.32 \\
7.25 \\
7.37 \\
7.41 \\
\overline{7.33} \\
7.44 \\
7.39 \\
7.37 \\
7.28 \\
\overline{7.31}\end{array}$ & $\begin{array}{l}48 \\
55 \\
59 \\
38 \\
52 \\
39 \\
45 \\
47 \\
\frac{57}{62}\end{array}$ & $\begin{array}{r}7.3 \\
8.0 \\
5.0 \\
12.3 \\
8.9 \\
6.0 \\
6.8 \\
7.8 \\
6.7 \\
8.4 \\
5.6 \\
4.4 \\
3.8\end{array}$ & \begin{tabular}{r|}
7.1 \\
8.4 \\
6.5 \\
10.6 \\
8.8 \\
6.7 \\
6.0 \\
6.9 \\
5.1 \\
7.7 \\
6.0 \\
4.1 \\
4.2
\end{tabular} \\
\hline $\begin{array}{l}\text { Mean } \\
\text { S.E. }\end{array}$ & $\begin{array}{r}18.1 \\
\pm 0.9\end{array}$ & $\begin{array}{r}47.2 \\
\pm 1.7\end{array}$ & $\begin{array}{r}7.40 \\
\pm 0.02\end{array}$ & $\begin{array}{r}40 \\
\pm 3\end{array}$ & $\begin{array}{r}11.1 \\
\pm 0.8\end{array}$ & $\begin{array}{r}53.9 \\
\pm 4.5\end{array}$ & $\begin{array}{r}7.35 \\
\pm 0.02\end{array}$ & $\begin{array}{r}50 \\
\pm 3\end{array}$ & $\begin{array}{r}7.0 \\
\pm 0.6\end{array}$ & $\begin{array}{r}6.8 \\
\pm 0.5\end{array}$ \\
\hline
\end{tabular}

* See footnote Table Ib.

$\mathrm{CMRO}_{2}$ were significantly reduced. Furthermore, the CVR in these cases was 31 per cent greater than that noted for uncomplicated hypertensives although the elevations of MABP noted in both groups were similar. The presence of a more severe degree of arteriosclerosis, particularly of the arteriolar variety, would seem to explain the impaired cerebral circulation and metabolism where hypertension and arteriosclerosis coexist.

The present data failed to demonstrate any significant differences between the hypertensive arteriosclerotic group with and that without a history of cerebrovascular accidents or mental deterioration. A comparison of these data with those of Freyhan, Woodford, and Kety (2) for patients with psychoses secondary to hypertensive arteriosclerotic vascular disease also fails to show a clear difference :

\begin{tabular}{|c|c|c|c|c|}
\hline & $\underset{m m . H g}{\operatorname{MABP}}$ & $\begin{array}{c}\text { CBF } \\
\text { cc./100 } \\
\text { gm./min. }\end{array}$ & $\begin{array}{c}\mathrm{CMRO}_{2} \\
\text { cc./100 } \\
\text { gm./min. }\end{array}$ & $\begin{array}{c}\text { CVR } \\
\text { mm. Hg/cc.I I } \\
100 \mathrm{gm} . / \mathrm{min} .\end{array}$ \\
\hline $\begin{array}{l}\mathrm{n}: \\
\text { series: }\end{array}$ & $\begin{array}{l}136 \\
139\end{array}$ & $\begin{array}{l}42 \\
39\end{array}$ & $\begin{array}{l}2.8 \\
2.4\end{array}$ & $\begin{array}{l}3.3 \\
3.4\end{array}$ \\
\hline
\end{tabular}

It would therefore appear that their implication that a reduced $\mathrm{CBF}$ and $\mathrm{CMRO}_{2}$ is the basis for the origin of the mental symptoms in their patients is not valid. Their conclusion was based upon the comparison of results with values for young normal individuals rather than a control group composed of individuals with comparable hypertension and arteriosclerosis but without psychoses.

The validity of these, and other data derived by the application of the nitrous oxide method to the study of pathological states, is subject to the objection that increased contamination with extra-

TABLE Va

Group 5: Clinical and cerebral circulatory data*

\begin{tabular}{|c|c|c|c|c|c|c|c|c|c|}
\hline Subject & Age & Sex & Diagnosis & MABP & JVP & CBF & CVR & $\mathrm{CMRO}_{2}$ & R.Q. \\
\hline $\begin{array}{l}\text { B. B. } \\
\text { G. B. } \\
\text { C. H. } \\
\text { S. P. } \\
\text { D. B. } \\
\text { R. S. }\end{array}$ & $\begin{array}{l}38 \\
38 \\
46 \\
39 \\
45 \\
56\end{array}$ & $\begin{array}{l}\mathbf{F} \\
\mathbf{F} \\
\mathbf{F} \\
\mathbf{F} \\
\mathbf{F} \\
\mathbf{M}\end{array}$ & $\begin{array}{l}\text { Ess. hyp. } \\
\text { Ess. hyp. } \\
\text { Ess. hyp. } \\
\text { Ess. hyp. } \\
\text { Ess. hyp. } \\
\text { Ess. hyp. }\end{array}$ & $\begin{array}{l}133 \\
154 \\
160 \\
116 \\
132 \\
121\end{array}$ & $\begin{array}{l}120 \\
137 \\
160 \\
120 \\
107 \\
165\end{array}$ & $\begin{array}{l}50 \\
60 \\
47 \\
55 \\
67 \\
31\end{array}$ & $\begin{array}{l}2.5 \\
2.4 \\
3.2 \\
1.9 \\
1.9 \\
3.5\end{array}$ & $\begin{array}{l}3.1 \\
3.7 \\
3.3 \\
2.8 \\
3.7 \\
1.9\end{array}$ & $\begin{array}{l}0.98 \\
1.09 \\
0.77 \\
0.79 \\
0.81 \\
1.15\end{array}$ \\
\hline $\begin{array}{l}\text { Mean } \\
\text { S.E. }\end{array}$ & 44 & & & $\begin{array}{l}136 t \\
\pm 7\end{array}$ & $\begin{array}{r}135 \\
\pm 10\end{array}$ & $\begin{array}{r}52 \\
\pm 5\end{array}$ & $\begin{array}{c}2.6 \dagger \\
\pm 0.3\end{array}$ & $\begin{array}{r}3.1 \\
\pm 0.3\end{array}$ & $\begin{array}{r}0.93 \\
\pm 0.07\end{array}$ \\
\hline
\end{tabular}

* See footnote Table Ia.

$\uparrow$ Differs significantly from value for Group 1 , p $<.05$. 
TABLE vb

Group 5: Blood constituents*

\begin{tabular}{|c|c|c|c|c|c|c|c|c|c|c|}
\hline Subject & $\mathrm{AO}_{2}$ & $\mathrm{ACO}_{2}$ & $\mathrm{ApH}$ & $\mathrm{ApCO}_{2}$ & $\mathrm{vo}_{2}$ & $\mathrm{VCO}_{2}$ & $\mathrm{VpH}$ & $\mathrm{VpCO}_{2}$ & $(A-V) O_{2}$ & $(\mathrm{~A}-\mathrm{V}) \mathrm{CO}_{2}$ \\
\hline $\begin{array}{l}\text { B. B. } \\
\text { G. B. } \\
\text { C. H. } \\
\text { S. P. } \\
\text { D. B. } \\
\text { R. S. }\end{array}$ & $\begin{array}{l}15.2 \\
16.6 \\
16.5 \\
14.7 \\
16.2 \\
16.0\end{array}$ & $\begin{array}{l}48.6 \\
45.7 \\
54.9 \\
48.5 \\
52.2 \\
44.8\end{array}$ & $\begin{array}{l}7.44 \\
7.41 \\
7.38 \\
7.39 \\
7.41 \\
7.40\end{array}$ & $\begin{array}{l}37 \\
37 \\
47 \\
41 \\
42 \\
37\end{array}$ & $\begin{array}{r}9.1 \\
10.4 \\
9.5 \\
9.6 \\
10.7 \\
10.0\end{array}$ & $\begin{array}{l}54.6 \\
52.4 \\
60.3 \\
52.6 \\
56.6 \\
51.7\end{array}$ & $\begin{array}{l}7.35 \\
7.33 \\
7.35 \\
7.31 \\
7.33 \\
7.36\end{array}$ & $\begin{array}{l}47 \\
49 \\
52 \\
51 \\
51 \\
43\end{array}$ & $\begin{array}{l}6.1 \\
6.2 \\
7.1 \\
5.1 \\
5.5 \\
6.0\end{array}$ & $\begin{array}{l}6.0 \\
6.7 \\
5.4 \\
4.0 \\
4.4 \\
6.9\end{array}$ \\
\hline $\begin{array}{l}\text { Mean } \\
\text { S.E. }\end{array}$ & $\begin{array}{r}15.9 \\
\pm 0.3\end{array}$ & $\begin{array}{r}49.1 \\
\pm 1.6\end{array}$ & $\begin{array}{r}7.41 \\
\pm 0.01\end{array}$ & $\begin{array}{r}40 \\
\pm 2\end{array}$ & $\begin{array}{r}9.9 \\
\pm 0.3\end{array}$ & $\begin{array}{r}54.7 \\
\pm 1.3\end{array}$ & $\begin{array}{r}7.34 \\
\pm 0.01\end{array}$ & $\begin{array}{r}49 \\
\pm 1\end{array}$ & $\begin{array}{r}6.0 \\
\pm 0.3\end{array}$ & $\begin{array}{r}5.6 \\
\pm 0.5\end{array}$ \\
\hline
\end{tabular}

* See footnote Table Ib.

cerebral blood may occur in the sampling from the internal jugular bulb. No direct studies of this point other than for normals (9) are available. However, the appearance of the curves plotted from blood nitrous oxide contents did not suggest appreciable contamination. Also of great importance is the fact that the nitrous oxide method measures blood flow per $100 \mathrm{gm}$. of perfused brain. In the presence of significant areas of infarcted brain, the total cerebral blood flow would be reduced to a greater extent than might be assumed on the basis of data indicating flow per $100 \mathrm{gm}$. of perfused brain.

A good correlation between a reduced CBF and a reduced $\mathrm{CMRO}_{2}$ has been noted in the present study (correlation coefficient 0.75 ). The possibility that the decreased CBF is secondary to the decreased $\mathrm{CMRO}_{2}$ may be mentioned but is unlikely. Alternative, but not mutually exclusive, explanations of this phenomenon are: 1) A decreased availability of oxygen because of a decreased $\mathrm{CBF}$ causes the reduced $\mathrm{CMRO}_{2} ; 2$ ) In arteriosclerotic vascular disease there is a diminished capillary interface or a diminished capillary permeability for gaseous exchange; 3 ) There is a decreased need for oxygen by the brain occurring independently but concurrently with the drop in CBF. Studies designed to test the ability of arteriosclerotics to maintain a constant $\mathrm{CMRO}_{2}$ in the face of induced reductions in CBF, and in vitro studies of brains of hypertensive arteriosclerotic animals offer avenues for further investigation of this relationship.

The occurrence of levels of cerebral oxygen consumption below those usually found in diabetic coma (10) or pentothal narcosis (11) has been observed in conscious, alert individuals. Such ob- servations emphasize the difficulties encountered in attempting to correlate levels of consciousness or mental activity with cerebral oxygen consumption. Although it is probably true that the cerebral oxygen consumption of an unconscious individual is lower than that particular individual's cerebral oxygen consumption in the conscious state, there appears to be no generally applicable absolute value for cerebral oxygen consumption which is characteristic of the presence of consciousness or unconsciousness.

\section{CONCLUSIONS}

1. Cerebral circulatory studies have been carried out on 54 individuals for the purpose of determining the effects of aging, arteriosclerosis, and hypertension upon the cerebral blood flow and metabolism.

2. Aging, arteriosclerosis unaccompanied by hypertension, and hypertension unaccompanied by arteriosclerosis were not found to reduce significantly the cerebral blood flow and metabolism.

3. The occurrence of hypertension and arteriosclerosis together was found to be accompanied by significant reductions of cerebral blood flow and oxygen consumption. This is ascribed to a greater severity of the arteriosclerosis, particularly of the arteriolar variety, in these cases.

4. No greater decreases of the cerebral blood flow and oxygen consumption were found in patients with clinical cerebrovascular disease when compared to values for similar patients without such manifestations.

5. Hypertension emerged from the present study as the one readily definable factor in predicting the occurrence of a reduced cerebral blood 
flow and oxygen consumption in arteriosclerotic individuals.

\section{REFERENCES}

1. Kety, S. S., and Schmidt, C. F., The nitrous oxide method for the quantitative determination of the cerebral blood flow in man: theory, procedure and normal values. J. Clin. Invest., 1948, 27, 476.

2. Freyhan, F. A., Woodford, R. B., and Kety, S. S., Cerebral blood flow and metabolism in psychoses of senility. J. Nerv. \& Ment. Dis., 1951, 113, 449.

3. Scheinberg, P., Cerebral blood flow in vascular disease of the brain, with observations on the effects of stellate ganglion block. Am. J. Med., 1950, 8, 139.

4. Fazekas, J. F., Alman, R. W., and Bessman, A. N., Cerebral physiology of the aged. Am. J. M. Sc., 1952, 223, 245.

5. Kety, S. S., Hafkenschiel, J. H., Jeffers, W. A., Leopold, I. H., and Shenkin, H. A., The blood flow, vascular resistance, and oxygen consumption of the brain in essential hypertension. J. Clin. Invest., 1948, 27, 511.

6. Peters, J. P., and Van Slyke, D. D., Quantitative Clinical Chemistry, Vol. II, Methods. Williams \& Wilkins, Baltimore, 1932.

7. Rosenthal T. B., The effect of temperature on the pH of blood and plasma in vitro. J. Biol. Chem., 1948, 173, 25.

8. Shenkin, H. A., Novack, P., and Bortin, L., Studies of the interrelationships of the cerebral blood flow, cerebrospinal fluid pressure, and jugular venous pressure. In preparation.

9. Shenkin, H. A., Harmel, M. H., and Kety, S. S., Dynamic anatomy of the cerebral circulation. Arch. Neurol. \& Psychiat., 1948, 60, 240.

10. Kety, S. S., Polis, B. D., Nadler, C. S., and Schmidt, C. F., The blood flow and oxygen consumption of the human brain in diabetic acidosis and coma. J. Clin. Invest., 1948, 27, 500.

11. Kety, S. S., Circulation and metabolism of the human brain in health and disease. Am. J. Med., 1950, 8, 205.

\section{SPECIAL NOTICE TO SUBSCRIBERS}

Post Offices will no longer forward the Journal when you move.

Please notify The Journal of Clinical Investigation, Business Office, 622 West 168th Street, New York 32, N. Y. at once when you have a change of address, and do not omit the zone number if there is one. 